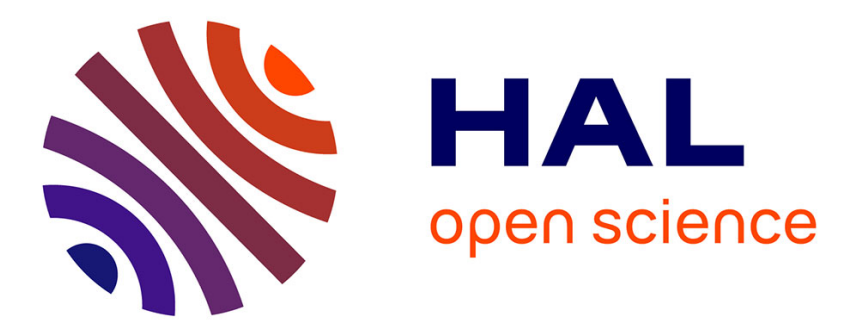

\title{
Integration of gradient based and response surface methods to develop a cascade optimisation strategy for Y-shaped tube hydroforming process design Rosa Di Lorenzo, Giuseppe Ingarao, Francisco Chinesta
}

\section{- To cite this version:}

Rosa Di Lorenzo, Giuseppe Ingarao, Francisco Chinesta. Integration of gradient based and response surface methods to develop a cascade optimisation strategy for Y-shaped tube hydroforming process design. Advances in Engineering Software, 2010, 41 (2), pp.336-348. 10.1016/j.advengsoft.2009.06.010 . hal-01006987

\section{HAL Id: hal-01006987 \\ https://hal.science/hal-01006987}

Submitted on 24 Apr 2017

HAL is a multi-disciplinary open access archive for the deposit and dissemination of scientific research documents, whether they are published or not. The documents may come from teaching and research institutions in France or abroad, or from public or private research centers.
L'archive ouverte pluridisciplinaire HAL, est destinée au dépôt et à la diffusion de documents scientifiques de niveau recherche, publiés ou non, émanant des établissements d'enseignement et de recherche français ou étrangers, des laboratoires publics ou privés. 


\title{
Integration of gradient based and response surface methods to develop a cascade optimisation strategy for Y-shaped tube hydroforming process design
}

\author{
Rosa Di Lorenzo ${ }^{\mathrm{a}}$, Giuseppe Ingarao ${ }^{\mathrm{a}}$, Francisco Chinesta ${ }^{\mathrm{b}}$ \\ ${ }^{a}$ Università di Palermo, Dipartimento di Tecnologia Meccanica, Produzione e Ingegneria Gestionale, viale delle Scienze 90128, Palermo, Italy \\ ${ }^{\mathrm{b}}$ EADS Corporate Foundation International Chair, Ecole Centrale de Nantes, 1 rue de la Noe, BP 92101, F-44321 Nantes cedex 3, France
}

\begin{abstract}
In the last years a strong research effort was produced in order to develop and design new forming technologies able to overcome the typical drawbacks of traditional forming operations. Among such new technologies, hydroforming proved to be one of the most promising. The design of tube hydroforming operations is mainly aimed to prevent bursting or buckling occurrence and such issues can be pursued only if a proper control of both material feeding history and internal pressure path during the process is performed.

In this paper, a proper optimisation strategy was developed on Y-shaped tube hydroforming process which is characterized by a quite complex process mechanics with respect to axi-symmetric tube hydroforming operations. The design procedure was aimed to properly calibrate the internal pressure histories. The basic idea, in this paper, is to integrate a steepest descent method with a moving least squares approach in order to reach the optimal internal pressure curve in the hydroforming of an Y-shaped steel tube. Thus, a cascade optimisation procedure was implemented which consisted of two optimisation steps: the former is focused on the application of a steepest descent method, the latter is based on a response surface approach utilising a moving least squares approximation. The cascade procedure was driven by the will to reduce the total number of numerical simulations necessary to reach the optimum with respect to other optimisation methods.
\end{abstract}

\author{
Keywords: \\ Tube hydroforming \\ Steepest descent method \\ Response surface method \\ Moving least squares \\ Cascade optimisation
}

\section{Introduction}

In the last years a strong research effort was produced in order to develop and design new forming technologies able to overcome the typical drawbacks of traditional forming operations. Among such new technologies, hydroforming proved to be one of the most promising. On the other hand, also a great interest in optimisation algorithms aimed to design forming processes was demonstrated by many researches.

Actually, in metal forming problems optimisation, several approaches were developed [1]: some approaches are characterized by a certain number of iterations of an iterative algorithm (usually gradient based) which are stopped when convergence is reached. Generally, such algorithms are interfaced with numerical simulation aimed to solve a certain number of direct problems necessary to calculate objective function gradients. Such algorithms may lead to local minima instead of global ones even if many of these algorithms are very efficient since few iterations are necessary to reach technologically satisfying results [2-8].
A totally different approach is based on intelligent techniques, for instance, genetic algorithms found a lot of applications [9-14] even if they generally require a high number of objective function evaluations. Some authors presented also some approaches which are based on the possibility to adapt the finite element analysis by some proper adaptive algorithms aimed to optimise some time dependent variables of a given process along the numerical simulations. Of course such approaches imply the possibility to manage the source code of a finite element software [15-17].

Maybe, one of the most effective approaches presented in the recent years in the technical literature is founded on response surface methodology [18]. Response surface methods (RSM) are based on approximation of a given objective function to be optimised through a set of points belonging to the domain of variation of the independent variables the function itself depends on.

Some works are focused on the application of RSM in sheet metal forming aimed to reduce the number of numerical simulations [19-23]: some authors used surrogate models and response surfaces in order to optimise a stamping operation for an automotive component [20] while other authors focused on springback effects control through RSM based approaches [21,22]. Another interesting approach concerns the sheet metal flanging process [23]. Actually, the main drawback of such method is the number of direct 
problem to be solved in order to reach good function approximations. A very interesting aspect in RSM application regards the possibility to build response surfaces basing on moving least squares approximations (MLS) by utilising a moving region of interest $[19,24]$. MLS are commonly used in mesh free methods as well as in many computational mechanics applications [25-29]. The MLS approach was also utilised in metal forming problems such as in optimising complex stamping operation even for industrial automotive cases: in particular the authors investigated the possibility to exploit MLS peculiarities in order to reach a high quality stamping part [19].

As tube hydroforming optimisation is concerned it has to be underlined that the basic idea in hydroforming is to replace costly dies with the action of a pressurised fluid on sheets or tubes [30]. The main result in the application of such technology is the reduction of tooling costs, nevertheless, also an improvement in process flexibility is gained with respect to traditional processes; in fact, a lower number of deformation stages are usually necessary to reach even complex formed shapes. Maybe, tube hydroforming is the principal example of hydroforming technologies advantages. Actually, tube hydroforming avoids the sequence of stamping and welding operations which characterizes the traditional tube production. In tube hydroforming processes the simultaneous actions of a fluid under pressure and a mechanical tool providing material feeding can lead to complex shape components characterized by good mechanical properties. The design of tube hydroforming operations is mainly aimed to prevent bursting or buckling occurrence and such issues can be pursued only if a proper control of both material feeding history and internal pressure path during the process is performed.

What is more, the production of T or Y-shaped tubes generally requires also the control of a counterpunch action with the aim to reduce thinning in tube bulged zones. In operations aimed to obtain Y-shaped tubes material feeding action is provided by two punches whose strokes have to be generally regulated in different ways.

The optimisation of tube hydroforming processes can be generally formulated as an optimisation problem in which some design variables have to be optimised in order to minimise a fixed objective function.

In this field, many studies were presented: some papers present integrations between numerical simulations and analytical or statistical procedures $[17,31]$; other researches investigated three dimensional processes with the aim to improve final part quality [32]. Many approaches concern the design of loading paths by numerical methods [33], while others utilised artificial intelligence to this aim $[34,35]$. The authors developed a wide research project on tube hydroforming optimisation even concerning three dimensional T-shaped tube hydroforming operations [36]; as Y-shaped tube hydroforming processes are concerned, some authors analysed their calibration with an integrated numerical-experimental approach [6].

The authors experienced the effectiveness of gradient based optimisation procedures, namely of steepest descent method, in designing the typical process parameters in tube hydroforming and the experimental validations supported such effectiveness [37]. Nevertheless, such procedure could lead to local optima instead of global ones. Moreover, a certain difficulty can arise in dealing with constraints to be taken into account during optimisation iterations.

In Y-shaped tube hydroforming design field, some authors presented the results of the application of Augmented Lagrangian and response surface methods for loading paths optimisation [38]. Such results prove that a significant number of numerical simulations are necessary to reach good results in terms of final product shape; namely the application of ALM led to a total number of numerical simulations which was higher than 150 .
Other applications of optimisation strategies, such as the utilisation of Sequential Approximate Optimization (SAO) algorithm, to tube hydroforming optimisation cases were presented but even in this cases more than 100 simulations were developed [1].

Moreover, the possibility to exploit the capabilities of different optimisation methods in an integrated methodology was investigated in recent years [39]. The authors have recently applied a decomposition approach, based on gradient techniques, in order to analyse a Y-shaped tube hydroforming operation as the one here studied [40].

Following the above considerations and the analysis of the technical literature, the authors propose an optimisation strategy focused on the utilisation of few design variables to be optimised to reach good performances on the chosen objective function. More in details, the proposed approach is based on the considerations discussed in the following.

- There is no one single robust and "correct" technique to solve a given optimisation problem; on the contrary the exploitation of the main advantages of different methods may be a promising approach.

- Gradient techniques, such as steepest descent method, are simple to be implemented and they work very well in identifying a sort of "optimum region" i.e. a region in the neighbourhoods of the real optimum.

- Gradient techniques allow a strong objective function reduction towards minimisation even starting from initial design variables values which are very far from real optimum.

- Gradient techniques are affected by some drawbacks such as a difficulty to converge in close proximity of the optimum (they risk to remain trapped into local optima) and a strong sensitivity to finite element code approximation in solving the necessary direct problems.

- Response surface methods work quite well in the neighbourhoods of the real optimum and the can be easily implemented if a reduced number of variables is taken into account.

- Moving least square approximations provides more accurate response surfaces when irregular grids of data have to be managed. This advantage allows, for instance, the reuse of points already available within the design variables domain thus reducing the required computational effort.

In particular, in this paper, an optimisation strategy was developed on Y-shaped tube hydroforming process which is characterized by a quite complex process mechanics with respect to axisymmetric tube hydroforming operations and even with respect to T-shaped tube ones. The design procedure was aimed to properly calibrate the internal pressure histories. Such aim was pursued by implementing a cascade optimisation procedure which consisted of two optimisation steps [41,42]: the former is focused on the application of a steepest descent method to reach an optimal solution which is in the neighbourhood of the actual optimum. The latter step of the optimisation process is based on a RSM utilising moving least squares approximation. Such final step is aimed to fine-tune the results in order to reach an optimal solution which is more satisfactory and in close proximity to the real optimum.

The basic idea, in this paper, is to integrate a steepest descent method with a MLS approach in order to associate and to exploit the advantages of both the utilised techniques. Moreover, the proposed approach also aims to minimise the number of numerical simulations necessary to accomplish the optimisation goals.

In the following sections the investigated problem will be presented as well as the optimisation procedure. Moreover, the results of the proposed optimisation strategy will be discussed and validated. Such validation was performed both by carrying out a sensitivity analysis at the varying of the starting solution and by 
comparing the obtained results with a fully second order approach. Namely, a procedure based on Central Composite Design technique to build up response surfaces was integrated with a sequential quadratic programming (SQP) approach to find the optimum of the investigated problem. The results of such validation proved the robustness of the obtained solution and of the proposed cascade procedure.

\section{The analysed Y-shaped tube hydroforming operation}

The proposed optimisation procedure aimed to design an hydroforming operation, namely, a tube hydroforming operation to manufacture an Y-shaped tube, Fig. 1 shows the utilised die geometrical details. The tube initial thickness was equal to $2 \mathrm{~mm}$ and an external diameter equal to $50 \mathrm{~mm}$; the tube material is a steel whose flow rule is expressed by the following equation that was experimentally identified:

$\sigma=653 \varepsilon^{0.253}$

The main aim of the design procedure implemented to optimise such process was to increase as much as possible the bulge height of the Y-region obtained at the end of the process also guaranteeing the minimisation of the final thinning on the tube. In other words, a double purpose was pursued: bursting danger reduction and bulge height maximisation.

In the Y-tube hydroforming operation design many factors influence the above mentioned objectives: internal pressure paths, punches velocity histories, counterpunch action etc. Some authors presented and demonstrated an effective way to calibrate the two punches actions [10]. In the presented application such hypothesis was followed namely, the punches velocity was kept constant but one of the punches has a total stroke which is half of the other one. Moreover, no counter punch action was taken into account. Thus, the only design variables which were considered are related to internal fluid pressure path. Namely, the pressure vs. process time curve shape was designed taking into account the available knowledge on the tube hydroforming process. Thus such shape consists of two linear paths with different slopes which is fully defined by three points coordinates (see Fig. 2).

In particular, the time instants corresponding to points $P 1, P 2$ and $P 3$ in Fig. 1 were fixed a priori according to a preliminary numerical analysis on the investigated process. As a consequence, the internal pressure path curve is fully defined if the three pressure values corresponding to the three chosen points are fixed.

According to these hypotheses the design problem here addressed can be translated into an optimisation problem characterized by three design variables (pressure values $P 1, P 2$ and $P 3$ ) and two conflicting goals. The former goal concerns both bursting prevention, the latter bulge height maximisation. The solution of such optimisation problem has to reach design variables values guaranteeing the optimisation of the two objectives. Such kind of problem may be solved through a multi-objective approach, considering the conflicting behaviour of the goals, and the authors have experi-

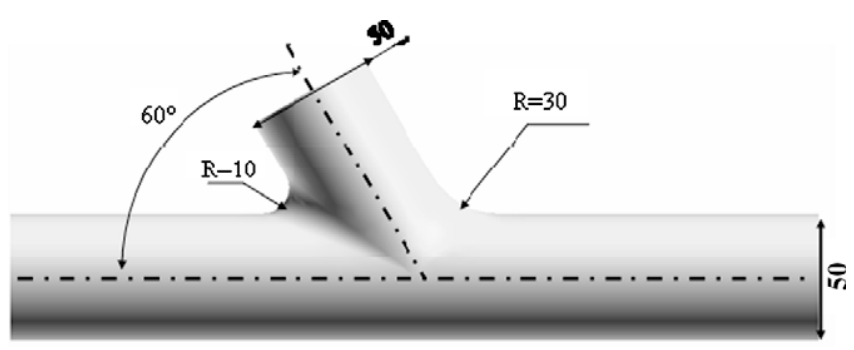

Fig. 1. The die geometry in the investigated process.

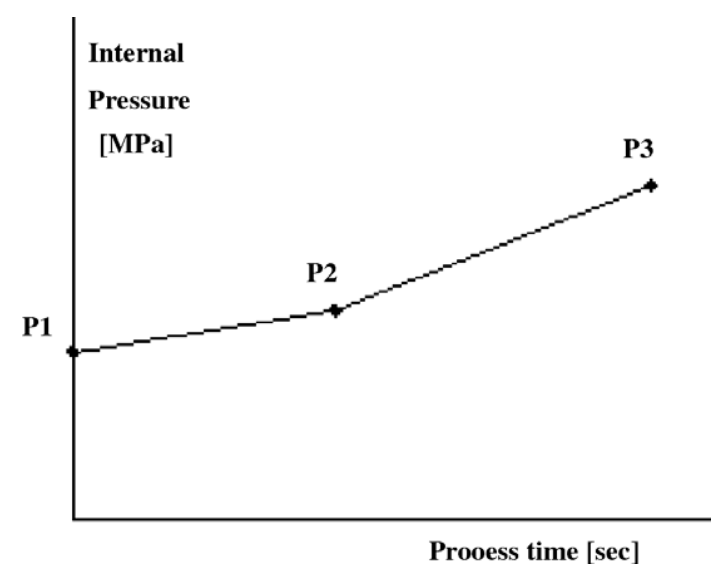

Fig. 2. The hypothesised pressure vs. process time curve shape.

enced the usefulness of such approaches [43]. Nevertheless, the problem here addressed was managed utilising an objective function consisting of two terms: the former is the maximum thinning on the tube walls ( $t \%$, which is related to the aim to reduce bursting danger) and the latter measuring the difference between the desired Y-region volume and the obtained one $(u$, measuring a sort of underfilling with respect to an objective Y-shape). This hypothesis is justified by the will to test the performances of the proposed cascade approach in terms of technological results applying it to a mono-objective problem. Moreover, the objective function formulation is performing from a technological point of view, since the goals to be pursued are effectively represented in this unique function (see Eq. (2)).

$f\left(x_{k}\right)=t \%+K u$

In this function, $K$ is the weight necessary to calibrate the contribution of the underfilling term. In the presented application two different values of the weight $K$ were considered in order to analyse the influence of the underfilling term. Such objective function has to be minimised to accomplish the design aims. It has to be underlined that the chosen objective function considers both fracture risk and underfilling; as the latter aspect is concerned the definition of the term $u$ in Eq. (2) allows to take into account and to evidence both wrinkling and buckling phenomena since such term is measured as a volumes difference.

As the optimisation procedure presented in this paper is considered, a starting solution had to be fixed. In other words, initial values of pressures $P 1, P 2$ and $P 3$ from which the optimisation procedure is started to search for different and optimal values.

In particular, the values in Table 1 were fixed as starting solution. Such values were chosen on the basis of the know-how acquired on the analysed process by preliminary numerical and experimental investigations aimed to determine the pressure levels which avoid buckling phenomena. In Fig. 3 the component obtained by utilising such pressure values is shown. In particular, the thinning distribution is reported as provided by the numerical code used to simulate the investigated operation all along the optimisation procedure (LS-DYNA).

As it can be observed, a critical wrinkling effect is obtained and the desired Y-shape is not reached. Moreover, a limited maximum thinning is achieved $(t \%=5.4 \%)$, thus the optimisation procedure should lead to a better final shape with no wrinkling defects and even with higher but not excessive thinning (i.e. not related to fracture occurrence). The optimisation procedure was applied in two different cases with respect to the value of the weight $K$ present in the objective function (see Eq. (2)): in case1 the weight $K$ is half of the one utilised in case 2 . What is more, a validation of the pro- 


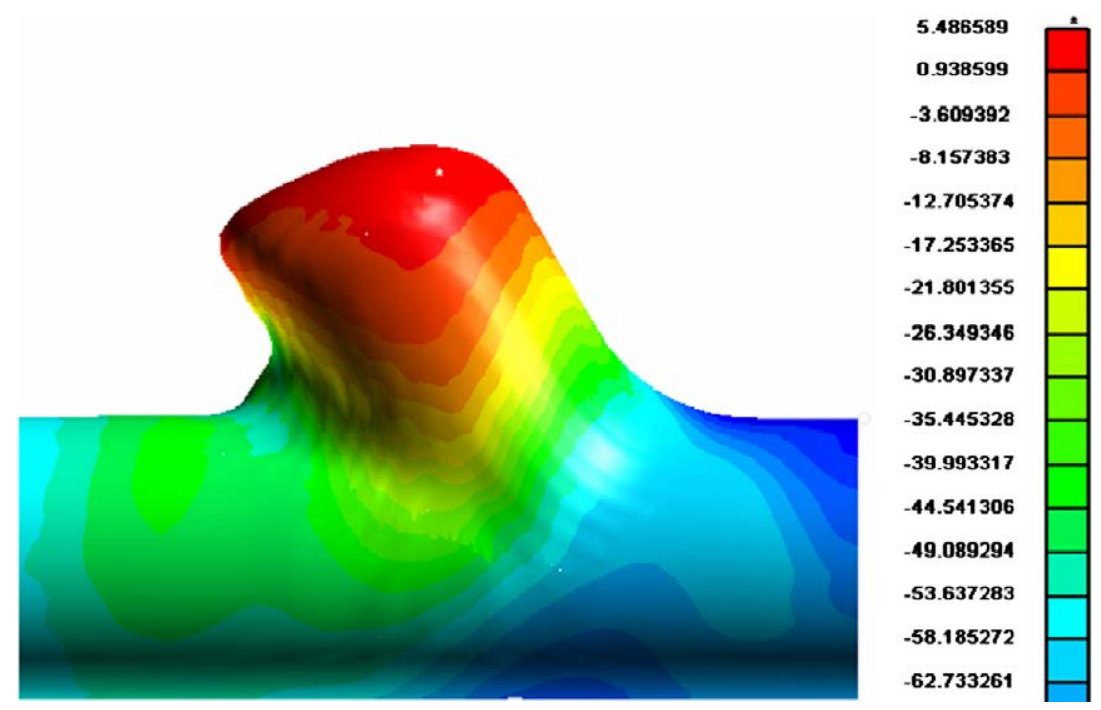

Fig. 3. The numerical result (thinning distribution) obtained by the initial pressure values.

cedure was performed both by a sensitivity analysis and by a comparison with a fully second order approximation. The sensitivity analysis was carried out to test the procedure results starting from different initial solutions and to prove the robustness of the method; the comparison with a different approach demonstrated the effectiveness of the cascade approach to reduce the computational effort.

\section{The utilised cascade optimisation procedure}

In this paper a cascade optimisation procedure was developed. In particular a mixed methodology was utilised consisting of two consecutive stages: the former uses a gradient based method while the latter is based on moving least squares approximation in a response surface approach.

The analytical formulation of a gradient based optimisation procedure can be summarised in the following steps ( $k$ denotes the method iteration number):

1. identify the design variables by a vector $\mathbf{x}_{k}$ at iteration $k$;

2. choose the initial values: $\mathbf{x}_{k} \in R^{n}$ with $k=0$;

3. calculate the gradient of the objective function $f\left(\mathbf{x}_{k}\right)$ : if the convergence is reached the algorithm can be stopped;

4. else calculate an updated value of the design variables $\mathbf{x}_{k+1}=\mathbf{x}_{k}+\alpha_{k} \mathbf{d}_{k}$ (where the scalar $\alpha_{k} \geqslant 0$ is called "step size" or "step length" at iteration $k$ and indicates the entity of design variables adjustment at iteration $k ; \mathbf{d}_{k}$ is the direction of movement i.e. the direction along which the objective function goes towards a minimum);

5. verify that $f\left(\mathbf{x}_{k+1}\right)<f\left(\mathbf{x}_{k}\right)$;

6. repeat steps 4 and 5 until convergence is reached.

Such general approach can be refined according to different techniques with respect both to the gradient calculation and to the definition of step size and step direction. The procedure proposed in this application was the steepest descent method. Such method is based on the hypothesis that if a minimum of the objective function is required then the search direction is given by the opposite of the function gradient; thus the following expression holds:

$\mathbf{x}_{k+1}=\mathbf{x}_{k}-\alpha_{k} \nabla f\left(\mathbf{x}_{k}\right)$
A finite difference method was utilised in order to calculate the gradient; fixing a perturbation of the design variables it was possible to calculate the gradient as follows:

$\frac{\delta f\left(\mathbf{x}_{k}\right)}{\varepsilon}=\frac{f\left(\mathbf{x}_{k}+\boldsymbol{\varepsilon}_{j}\right)-f\left(\mathbf{x}_{k}\right)}{\varepsilon}$
where $\boldsymbol{\varepsilon}_{j}$ is defined as $\left[\begin{array}{c}0 \\ 0 \\ \vdots \\ \varepsilon \\ \vdots \\ 0\end{array}\right]$ i.e. a column vector whose components are null except than the one of the $j$ th row which is equal to $\varepsilon$.

Thus, the calculation of the gradient required the evaluation of the objective function values for each value and for each perturbation of the design variables. Such evaluation of the objective function was obtained through the solution of direct problems i.e. numerical simulations aimed to evaluate the values assumed by the objective function at the varying of the design variables. As the step size evaluation is concerned, a line search procedure was utilised in order to determine the most performing value.

It is worth pointing out that gradient based methods are very effective if a rough optimal solution is searched; actually, they allow to reach satisfactory results on technological problems with a low computational effort but they can remain entrapped into local optima.

If a fine-tuning of the solution is needed gradient based approaches are not so advantageous due to convergence problems which could lead to an excessive number of direct problems to be solved. In particular, a steepest descent procedure decreases its performances if a narrow region around the optimum is explored along the method iterations.

For such reasons the basic idea of the proposed cascade procedure was to couple a steepest descent procedure with a response surface approach.

In particular, in the first step of the optimisation procedure a steepest descent method was implemented in order to identify the optimum "region", while in the latter step, a moving least squares approach allowed a better optimum definition with a significant improvement of the solution. In other words, the gradient based approach drives towards the optimum but the MLS approach finds it out. 
The steepest descent iterations were stopped when no further improvements of the objective function were possible and the obtained solution was utilised to implement a response surface method by building up a proper design of experiments (DOE) around the solution itself. The DOE design was driven by the will to reduce the number of points to be analysed (again with the main aim to limit the numerical simulations number).

Response surface methodologies aim to approximate the objective function by a polynomial function over the design variables space. The general scheme of the application of RSM consists in the design domain definition (i.e. the space spanned by the design variables also called region of interest) followed by the calculation

Table 1

Initial pressure values.

\begin{tabular}{lll}
\hline$P 1(\mathrm{MPa})$ & $P 2(\mathrm{MPa})$ & $P 3(\mathrm{MPa})$ \\
\hline 20 & 25 & 40 \\
\hline
\end{tabular}

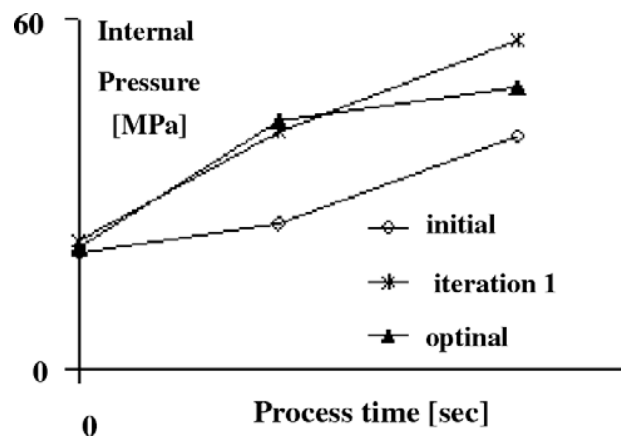

Fig. 4. The evolution of the steepest descent optimisation algorithm in case1.

Table 2

Optimal pressure values by the gradient based procedure in case1.

\begin{tabular}{lll}
\hline$P 1(\mathrm{MPa})$ & $P 2(\mathrm{MPa})$ & $P 3(\mathrm{MPa})$ \\
\hline 21 & 43 & 48 \\
\hline
\end{tabular}

of the objective function approximation in order to find out the optimum [13].

The convergence of the method is reached by rebuilding a restricted region of interest around the determined optimum and iterating the procedure to get a new function approximation. For an optimisation problem with $n$ design variables and indicating with $\mathbf{x} \in R^{n}$ the vector whose components are the design variables, the application of a response surface approach leads to an approximation $\bar{f}(\mathbf{x})$ of the objective function $f(\mathbf{x})$. Such approximation is possible on the basis of some values of the objective function known over the region of interest. This knowledge can be achieved by building up a DOE which has to be properly defined in order to get a good knowledge of the function but also to limit the number of experiments/simulations. Moving least squares approach proved to be very useful in exploring the design space by subsequent movements along consecutive minima determined on spanned and zoomed regions of interest $[12,26]$ and utilising also irregular grids of points belonging to the design space.

The first assumption in the application of RSM approach is that the approximated function can be written as:

$$
\bar{f}(\mathbf{x})=p^{T}(\mathbf{x}) a(\mathbf{x})
$$

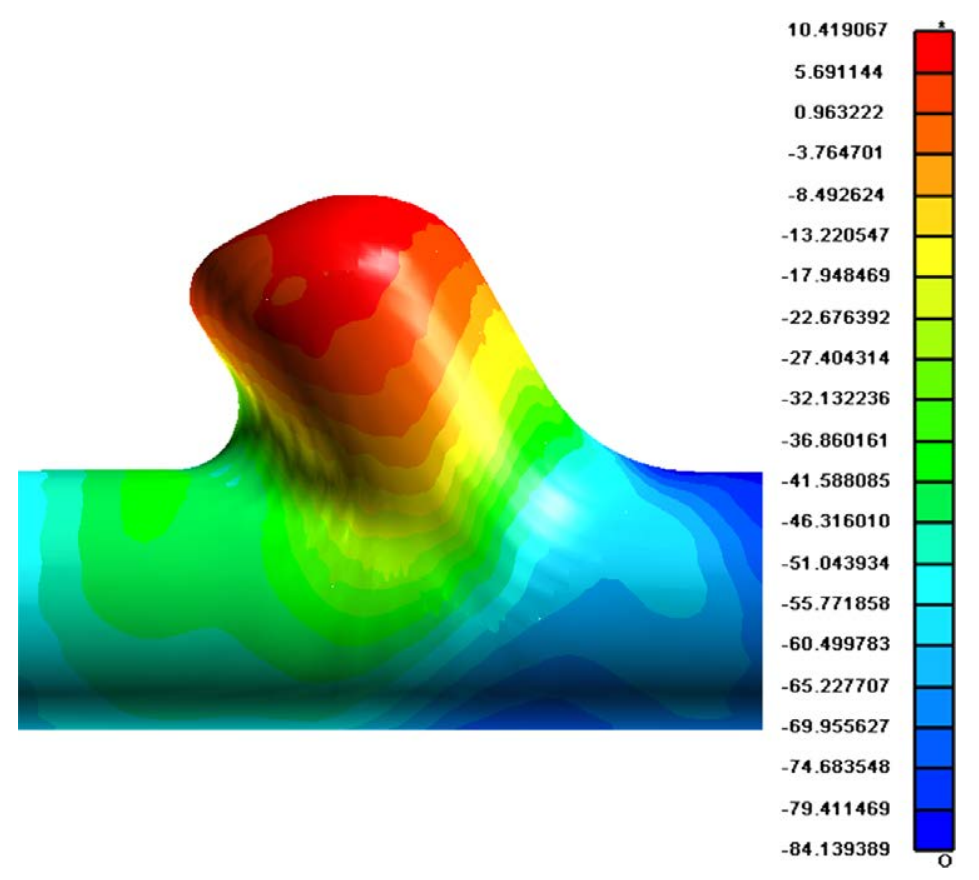

Fig. 5. The numerical simulation results (thinning distribution) corresponding to gradient based optimum in case1. 
Table 3

Optimal pressure values by the procedure in case $1 \mathrm{a}$.

\begin{tabular}{lll}
\hline$P 1(\mathrm{MPa})$ & $P 2(\mathrm{MPa})$ & $P 3(\mathrm{MPa})$ \\
\hline 21 & 43 & 48 \\
18.5 & 40 & 45 \\
23.5 & 46 & 45 \\
23.5 & 40 & 45 \\
18.5 & 46 & 45 \\
18.5 & 40 & 51 \\
23.5 & 46 & 51 \\
23.5 & 40 & 51 \\
18.5 & 46 & 51 \\
\hline
\end{tabular}

Table 4

Optimal pressure values by the procedure of case1a.

\begin{tabular}{lll}
\hline$P 1(\mathrm{MPa})$ & $P 2(\mathrm{MPa})$ & $P 3(\mathrm{MPa})$ \\
\hline 18.5 & 46 & 51
\end{tabular}

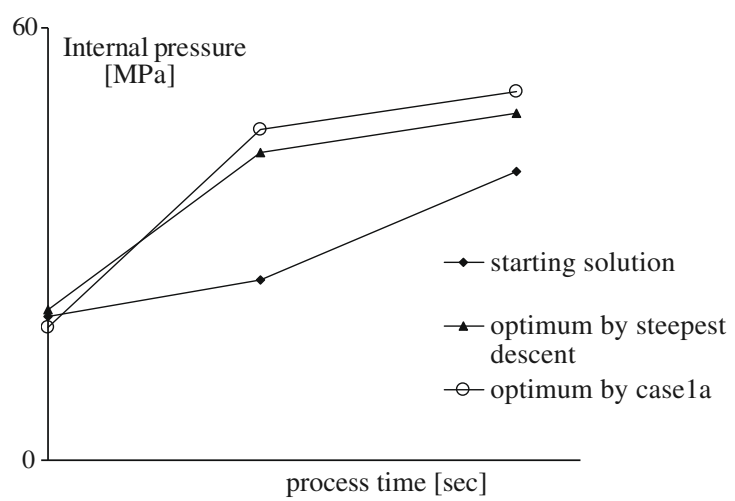

Fig. 7. Comparison between steepest descent optimum and the one of case1a.
Thus, the basis function $p(\mathbf{x})$ has to be defined through the following expression:

$p(\mathbf{x})=\left\langle\begin{array}{lllllllllll}1 & x_{1} & \cdots & x_{n} & x_{1} x_{2} & \cdots & x_{i} x_{i+1} & \cdots & \frac{x_{1}^{2}}{2} & \cdots & \frac{x_{n}^{2}}{2}\end{array}\right\rangle^{T}$

where $x_{1} \ldots \mathrm{x}_{n}$ are the components of vector $\mathbf{x}$.

As the coefficients $a(\mathbf{x})$ are regarded, they can be determined by minimising the error between actual and approximated values of the objective function. Such error can be written as:

$E(a)=\sum_{j=1}^{D} w\left(\left\|\mathbf{x}_{j}-\mathbf{x}\right\|\right)\left(p^{T}\left(\mathbf{x}_{j}-\mathbf{x}\right) a-f\left(\mathbf{x}_{j}\right)\right)^{2}$

being $D$ the total number of available values. Thus, the results of such minimisation provide:

$a(\mathbf{x})=A^{-1} B f$

In particular, the two matrices can be expressed as: $A=P W P^{T}$ and $B=P W$ indicating with $\mathbf{P}: \mathbf{P}=\left[\ldots p\left(\mathbf{x}_{j}-\mathbf{x}\right) \ldots\right]$ and with $W$ the following matrix:

$W=\left[\begin{array}{cccc}w\left(\mathbf{x}_{1}-\mathbf{x}\right) & & & 0 \\ & w\left(\mathbf{x}_{2}-\mathbf{x}\right) & & \\ & & \ddots & \\ 0 & & & w\left(\mathbf{x}_{D}-\mathbf{x}\right)\end{array}\right]$

The MLS application consists in choosing an initial design point $\mathbf{x}$, updating the coefficients $a(\mathbf{x})$ (see Eq. (8)) and iterating the procedure.

In this paper, the optimisation procedure implements the MLS after a steepest descent method. Thus, the starting point of MLS procedure is the optimum determined by steepest descent iterations. One of the aims in the application of such procedure was the reduction of the number of such direct problems. As a consequence the regions of interest analysed at each iteration of the MLS approach were built up also taking into account the possibility

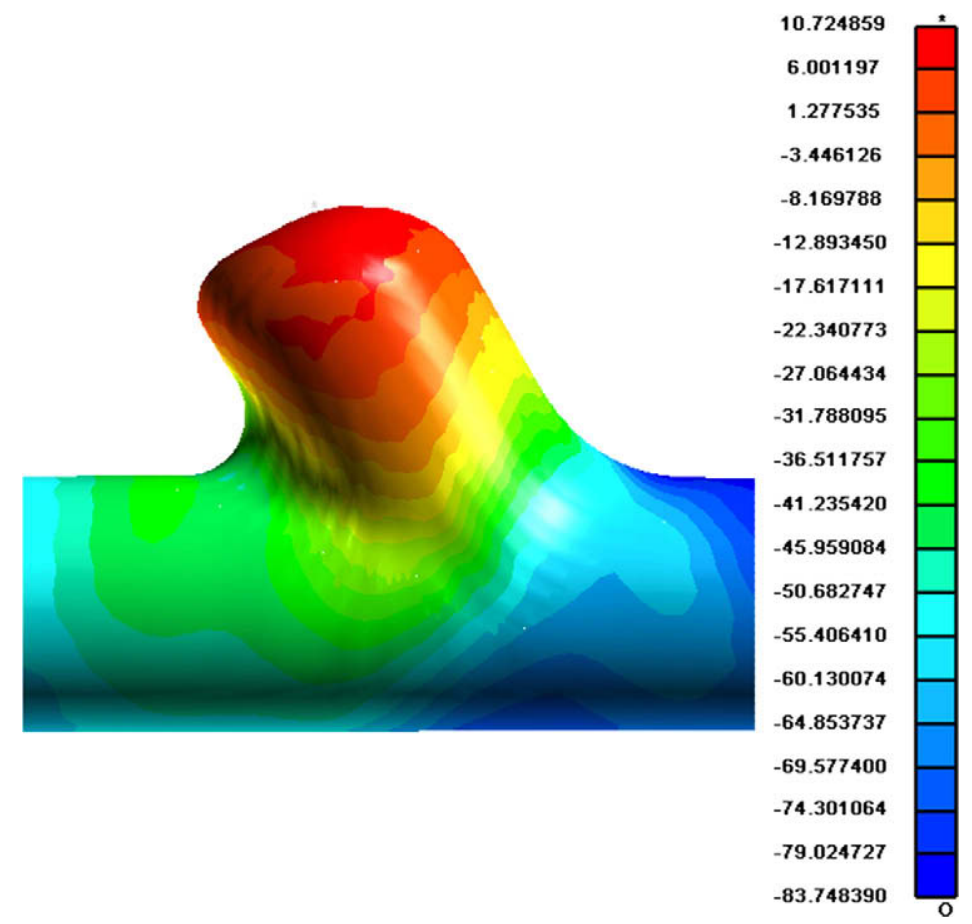

Fig. 8. The numerical simulation results (thinning distribution) corresponding to optimum in case $1 \mathrm{a}$. 
to "reuse" points yet available i.e. direct problems solved during steepest descent iterations preceding MLS application.

Following the MLS approach, the function $\bar{f}(\mathbf{x})$ was known and it was minimised in order to find the minimum of the obtained surface over the domain.

\section{Cascade optimisation of Y-shaped tube hydroforming}

As mentioned two cases were investigated. In the former (case 1$)$ the weight $K$ is half $(0.05)$ of the one (0.1) utilised in the latter (case2). The application of the cascade optimisation procedure to case 1 is discussed in the following.

The first step of the procedure, i.e. the application of a steepest descent method, consisted of three iterations. The evolutions of the algorithm are illustrated in Fig. 4 for case1.

Table 5

Optimal pressure values by the MLS procedure (with reused points) in case $1 \mathrm{~b}$.

\begin{tabular}{lll}
\hline$P 1(\mathrm{MPa})$ & $P 2(\mathrm{MPa})$ & $P 3(\mathrm{MPa})$ \\
\hline 19 & 49 & 50.5 \\
\hline
\end{tabular}

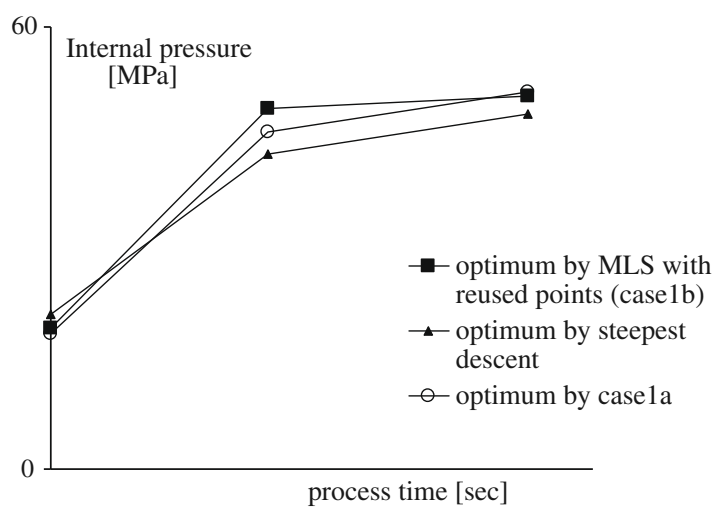

Fig. 9. Comparison among the different optima obtained in the cascade procedure for case1.
As already mentioned, in the neighbourhood of the expected minimum, the steepest descent algorithm is supposed not to perform well since its linear approximation would imply several iterations leading to narrow modifications of the design variables. In order to avoid such relevant computational effort, which, in turn, would not provide a significant improvement of the results, the steepest descent iterations were stopped when no significant change of the design variables were allowed; such stopping criterion implies that no significant improvement of the objective function can be reached. In other words, the modifications which can be provided by the steepest descent iterations are not technologically significant (i.e. the variations of pressure values would be of few MPa and such variations would not significantly change the process mechanics), thus no effects on the final solution can be expected. The optimal values of the three pressure variables, reached by the gradient based procedure, are illustrated in Table 2 (in the following such point is indicated with $A_{0}$ ) while Fig. 5 reports the numerical simulation results (thinning distribution) corresponding to such optimum values.

In such case a maximum thinning $t \%=10.4 \%$ is reached, which is quite satisfactory for the analysed material, and also a final Yshape closer enough to the desired one is obtained. Thus the obtained solution can be considered good from a technological point of view; nevertheless, an improvement can be expected. Thus, a DOE around the optimum shown in Table 2 was built up. Fig. 6 shows the shape of the utilised DOE which consists of only eight points around the current optimum (indicated with $A_{0}$ in Fig. 6).

Before proceeding with the optimisation workflow, a linear approximation was applied over the DOE illustrated in Fig. 6. This approximation (in the following indicated as case1a), obtained by a RSM approach, was performed with the aim to evidence its different prediction capability with respect to one subsequently obtained from a quadratic MLS approximation. Table 3 shows the values of the design variables of the utilised 8-points DOE in the case here addressed.

The proposed case1a led to a response surface whose minimisation provided a different optimal solution which is illustrated in Table 4 and compared with the one obtained by steepest descent steps in Fig. 7.

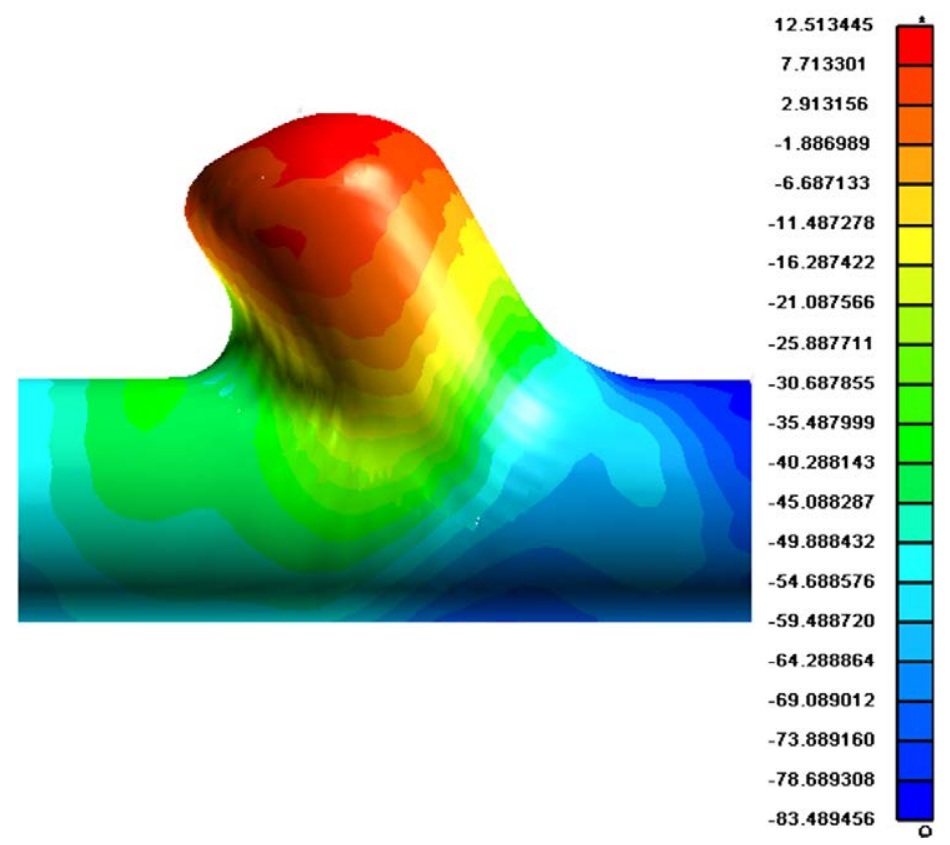

Fig. 10. The numerical simulation results (thinning distribution) corresponding to MLS optimum in case1b. 


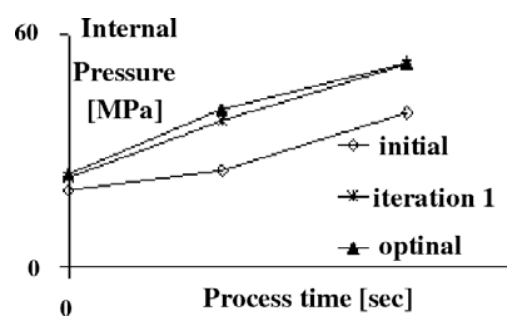

Fig. 11. The evolution of the steepest descent optimisation algorithm in case2.

Table 6

Optimal pressure values by the gradient based procedure in case2.

\begin{tabular}{lll}
\hline$P 1(\mathrm{MPa})$ & $P 2(\mathrm{MPa})$ & $P 3(\mathrm{MPa})$ \\
\hline 24 & 41 & 53 \\
\hline
\end{tabular}

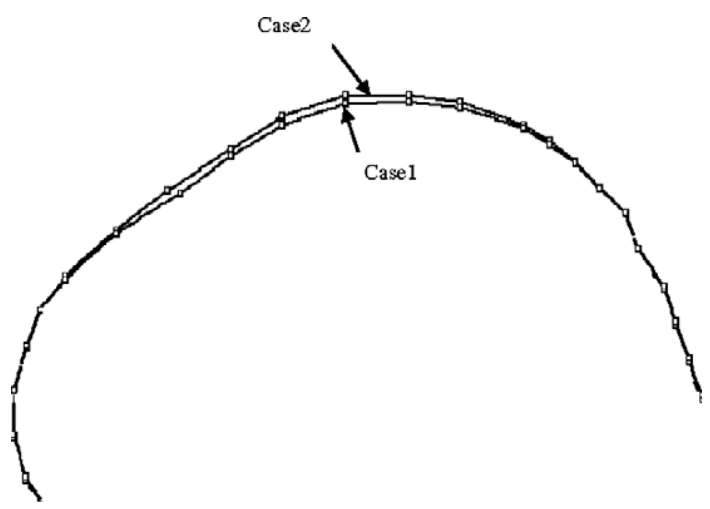

Fig. 12. The final profiles of the Y-shape obtained after steepest descent procedure in the two investigated cases.

As it can be noticed a variation is obtained by the case 1 a procedure as the optimal pressure curve is concerned; the objective function underwent a further reduction i.e. the procedure moves towards the actual minimum of the objective function itself. As expected, the optimal solution belongs to one of the boundaries of the chosen DOE; in fact, the response surface was an hyper plane which indicates a descending direction: the most performing point in that direction was the point illustrated in Table 4 which is on the DOE boundary.

Fig. 8 shows the thinning distribution for the new solution i.e. corresponding to the new optimised pressure curve. The maximum thinning in this case is a little bit higher than the one corresponding to the pressure curve optimised by the steepest descent method, in fact its value is about $10.7 \%$ but the underfilling is lower, as it can be noticed from the bulge zone height in Fig. 8 .

A different approach characterizes the proposed cascade optimisation procedure: after the steepest descent application, a DOE for MLS iterations was developed; such DOE was built including some points already known from the previous iterations of the steepest descent. The possibility provided by MLS approach to reuse points yet available, was exploited: this permitted to use a fully second order approximation of the objective function. The utilised DOE consisted of a total number of 16 points (case1b) and it is composed by the eight points previously utilised for the case1a and by eight points already available from the steepest descent procedure which belong to the region of interest identified by the 8-points DOE illustrated in Fig. 6. The quadratic approximation gave the better results and a further optimum was gained (see Table 5) which is the most performing one.

As it can be observed in Table 5 this new optimum can be considered, as expected, a fine-tuning of the previous solution proving that a cascade procedure leads towards more accurate results. Fig. 9 illustrates the different optimal pressure curves obtained in case1.

This final optimum allows a maximum thinning of about $12.5 \%$ and a quite good filling of the bulge zone (see Fig. 10), also leading to a further improvement of the objective function toward minimisation.

The same cascade procedure was implemented for case 2 in which as mentioned a higher $K$ is used in the objective function in order to give much weight to underfilling occurrence. In fact,

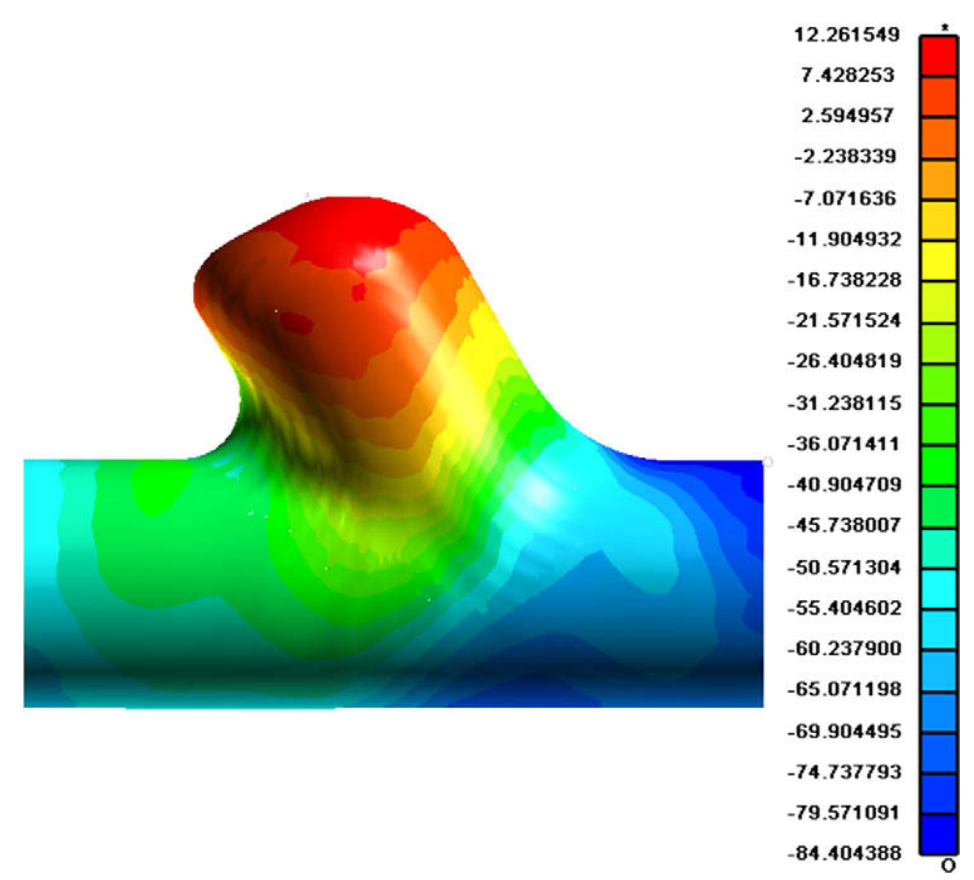

Fig. 13. The numerical simulation results (thinning distribution) corresponding to gradient based optimum in case 2 . 


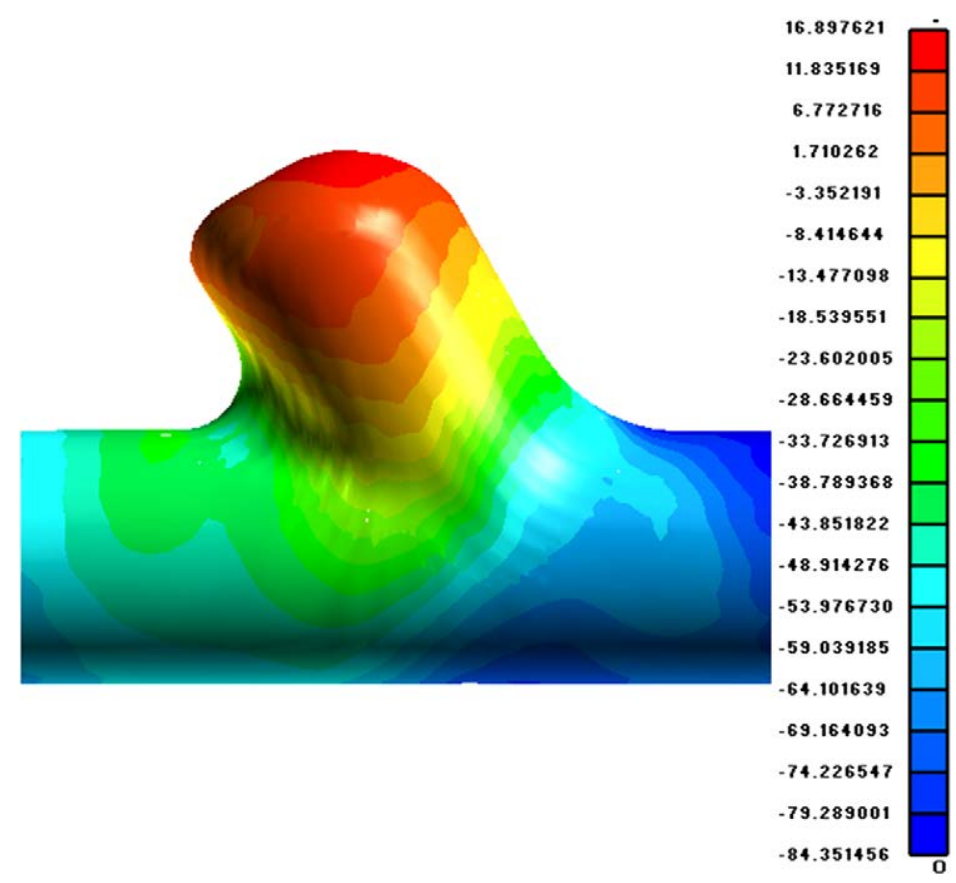

Fig. 14. The numerical simulation results (thinning distribution) corresponding to case2a.

in case 1 the results were very good but an improvement is desirable in terms of final bulge zone shape i.e. in terms of underfilling decrease. Actually, very satisfactory results were obtained in term of underfilling minimisation. As the steepest descent implementation is concerned, the evolutions toward the optimum are shown in Fig. 11 (also in this case 3 iterations were run).

Moreover, Table 6 shows the optimal values of the design variables obtained by the steepest descent method in case 2 .

In such case the optimisation procedure led to a maximum thinning equal to $12.3 \%$ which is slightly worse with respect to
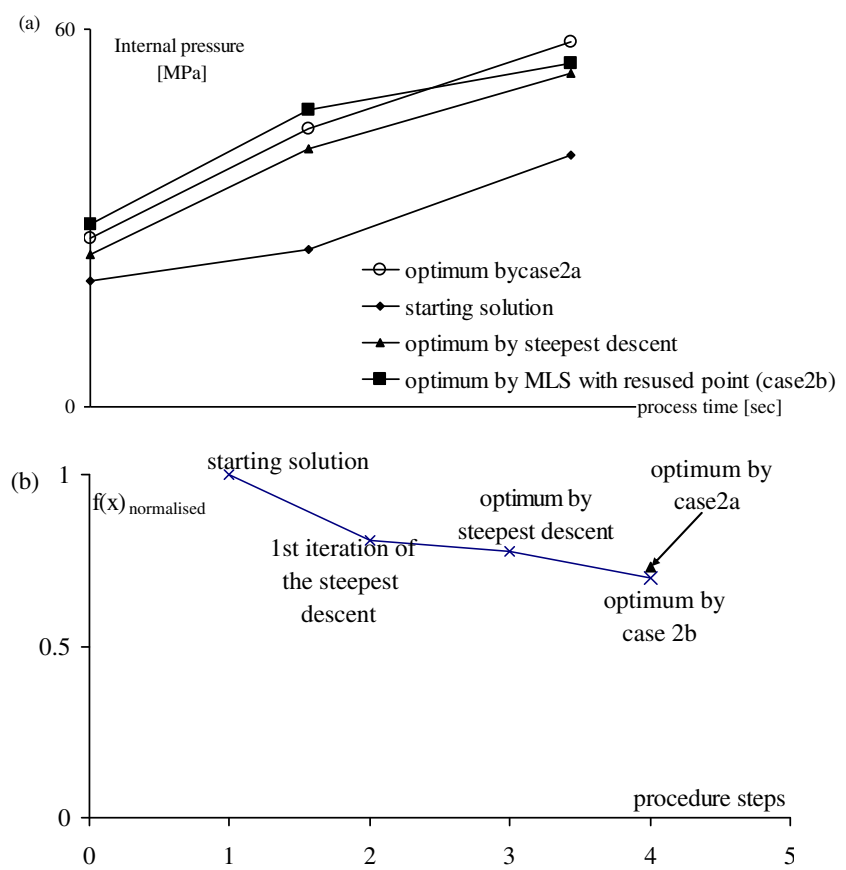

Fig. 15. Comparison among the different optima obtained in the cascade procedure for case2 (a) and objective function variations in the different steps of case2 (b). the previous case. On the other hand, a better result was obtained in terms of underfilling. In fact, in case2, the underfilling related term in the objective function has a stronger weight with respect to case1; thus, the gradient method provides an optimal solution which corresponds to better performance on underfilling. Actually, if the weight $K$ in the objective function is increased a higher bulge height with a worse thinning is expected. Fig. 12 shows a geometrical comparison of the final profiles of the Y-shape obtained in the two cases after the application of the steepest descent optimisation. As it can be noticed, an improvement of the final shape is reached with a higher final bulge height. Fig. 13 reports the numerical simulation (thinning distribution) results for case2.

Also in case 2 the cascade optimisation procedure was implemented by utilising again a DOE consisting of 8-points as the one illustrated in Fig. 6. Again, case2 was split in two different cases (case2a and case2b, respectively): in the former the 8-points DOE was utilised while in the latter a 13-points one was built by reusing some of the points available from the steepest descent iterations.

Fig. 14 illustrates the result of MLS in case2a. As it can be observed a maximum thinning of about $17 \%$ is reached with a good result in terms of underfilling.

Anyway, the best solution was obtained in case2b by implementing the reuse of previous points for the MLS approximation. In Fig. 15 the comparison of the different optima is shown together with the evolutions of the objective function in the different steps. Again the best solution is a fine-tuning of previous ones.

The optimal solution obtained in case $2 \mathrm{~b}$ is the most performing one all over the procedures. Actually, a quite good component was reached as both thinning and underfilling are regarded. Table 7

Table 7

Optimal pressure values by the MLS procedure (with reused points) in case $2 \mathrm{~b}$.

\begin{tabular}{lll}
\hline$P 1(\mathrm{MPa})$ & $P 2(\mathrm{MPa})$ & $P 3(\mathrm{MPa})$ \\
\hline 29 & 47 & 54.5 \\
\hline
\end{tabular}




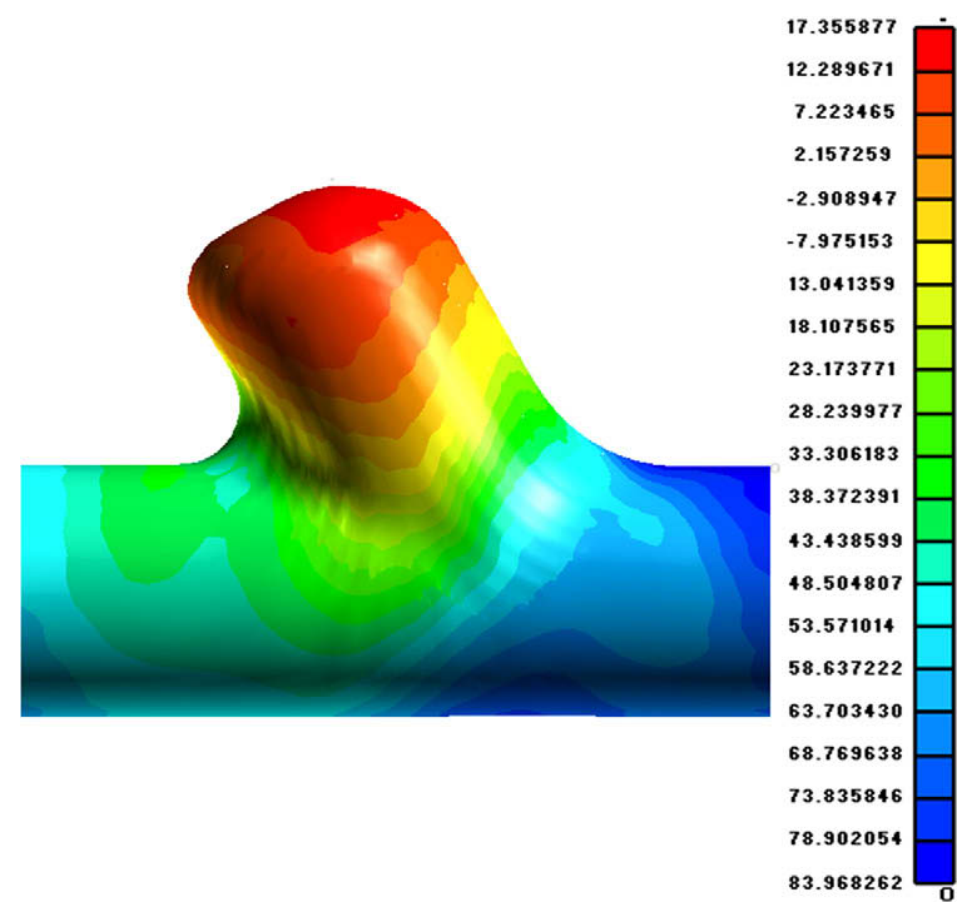

Fig. 16. The numerical simulation results (thinning distribution) corresponding to MLS optimum in case2b (with reused points).
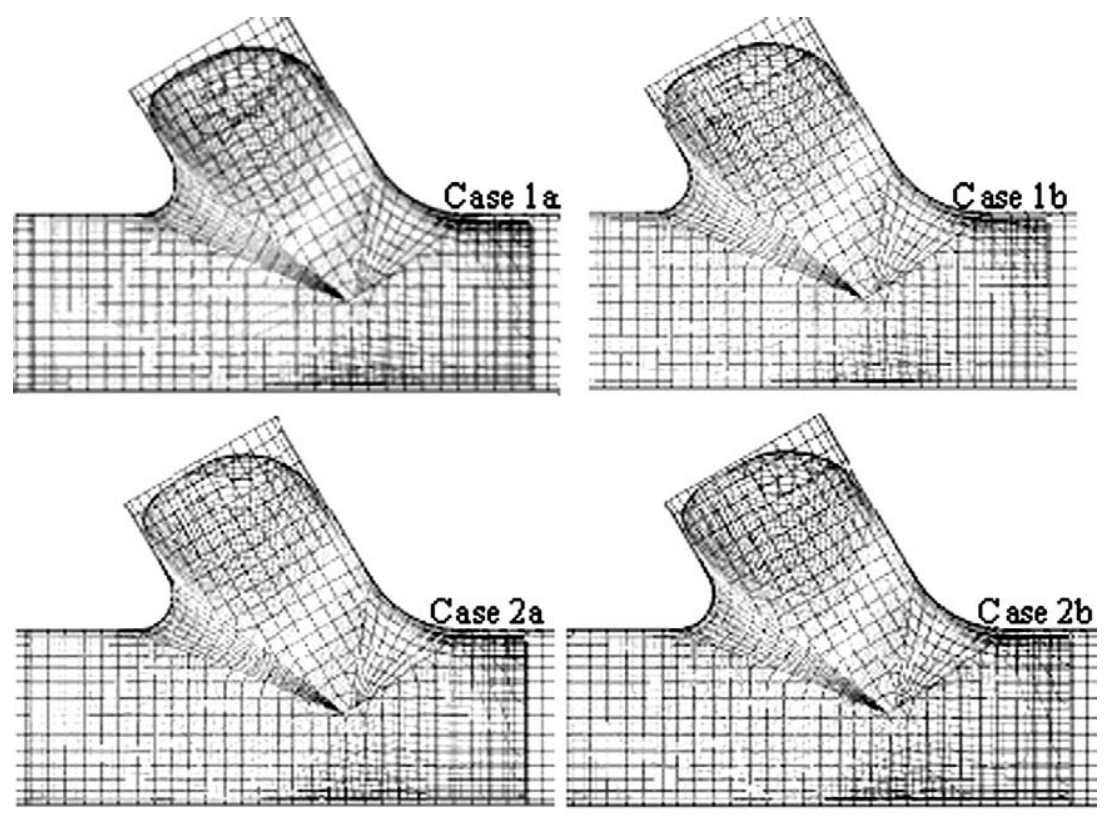

Fig. 17. The final shapes obtained in the four analysed cases.

summarises the optimal pressure values obtained by the most performing procedure.

Such solution allows to get a maximum thinning of $17.3 \%$ together with the lowest obtained value of underfilling i.e. with the best performances in terms of final bulged shape and, as a consequence with the lowest reached value of the objective function (which means that such results is the nearest to the actual minimum). Fig. 16 highlights the above mentioned results reporting the numerical thinning distribution and showing the obtained filling.

Moreover, in order to evidence the improvement obtained in terms of final component quality as bulged height and die filling are concerned, Fig. 17 shows the comparison among the final deformed shapes obtained in the investigated cases above presented. As it is clear, a rather significant improvement of the final shape was reached.

In order to understand the evolutions of the final profile Fig. 18 shows the comparison between the final shapes obtained in cases $1 \mathrm{~b}$ and $2 \mathrm{~b}$, respectively, highlighting the role of weight $K$ to drive towards better die filling. Fig. 19 highlights the profiles obtained for case 2 after steepest descent optimisation and also in case2a and $2 \mathrm{~b}$. Again such results confirm the evolution of the procedure towards a more accurate solution since a second order approximation gives more precise predictions. 


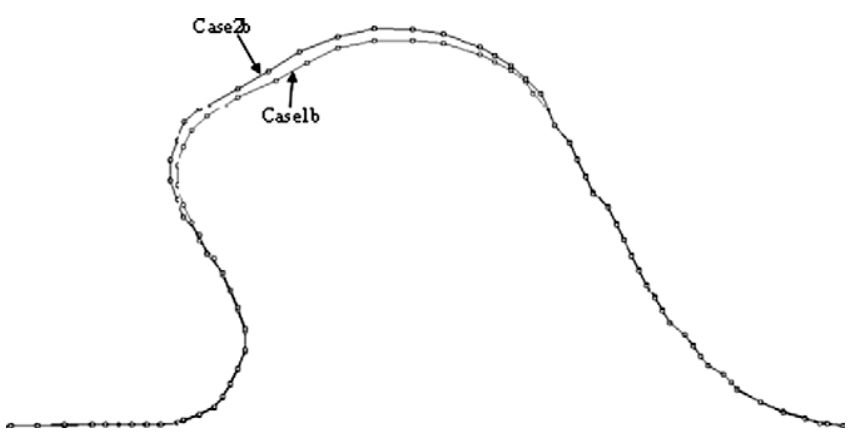

Fig. 18. The final profiles of the Y-shape obtained in cases $1 b$ and $2 b$.

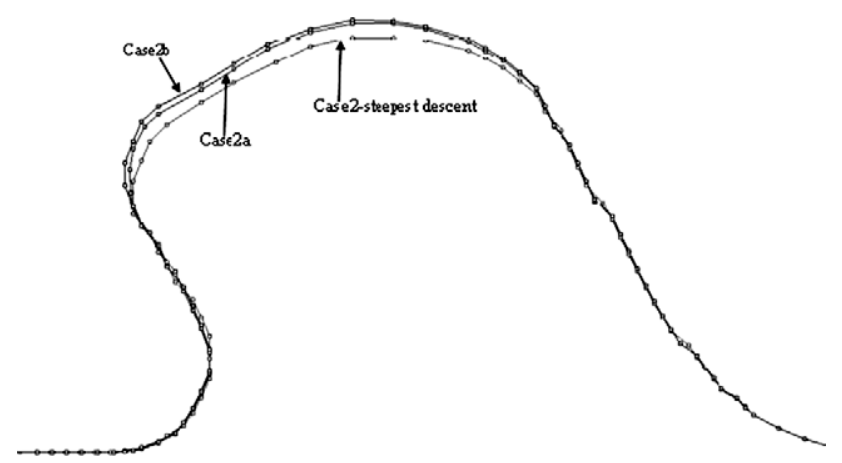

Fig. 19. The final profiles of the Y-shape obtained in case 2 .

\section{Validation of the procedure}

After the achievement of the best performing optimal solution through the cascade optimisation procedure, the robustness of the method was tested at the varying of the starting solution and also comparing the results with the ones derived from a fully second order approach.

As the former issue is concerned, the influence of the variation of the initial pressure curves was investigated. In this way, other three starting curves were utilised which are shown in Fig. 20 (starting solution 1 corresponds to the pressure values reported in Table 1).

As it can be observed, higher pressure levels were utilised with respect to the first starting solution since lower ones could determine buckling occurrence according to the preliminary numerical analysis developed on the analysed process. Moreover, the different starting solutions were chosen also with the aim to explore

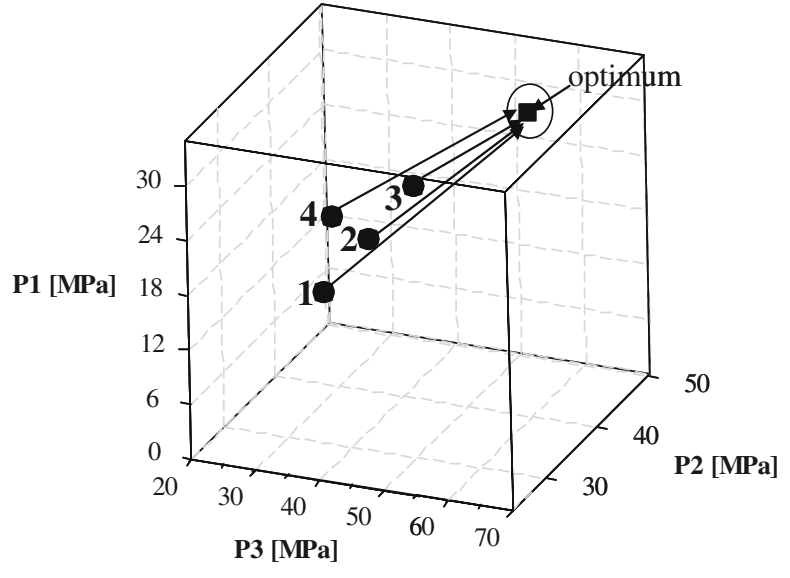

Fig. 21. The results robustness.

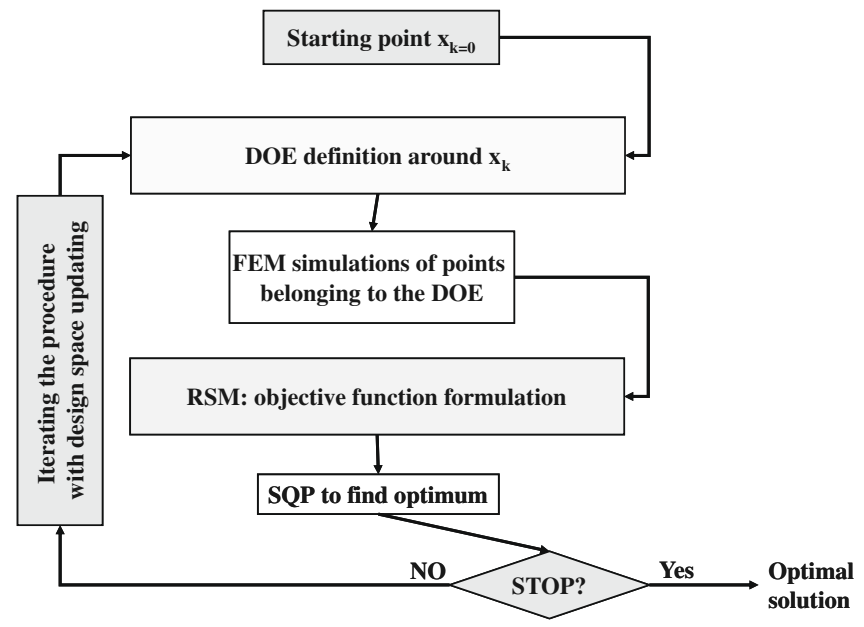

Fig. 22. The workflow of procedure of the fully second order approach.

the influence of the variation of all the three pressure values $(P 1$, $P 2$ and $P 3$ ). The procedure applied for all the starting solutions was the one developed for case $2 \mathrm{~b}$ since it was the most performing one. The results of the performed tests demonstrated the robustness of the reached optimum; in fact, as illustrated in Fig. 21 the same optimum is reached even if different starting solutions are utilised, which is a quite satisfactory result for the optimisation of the investigated process.

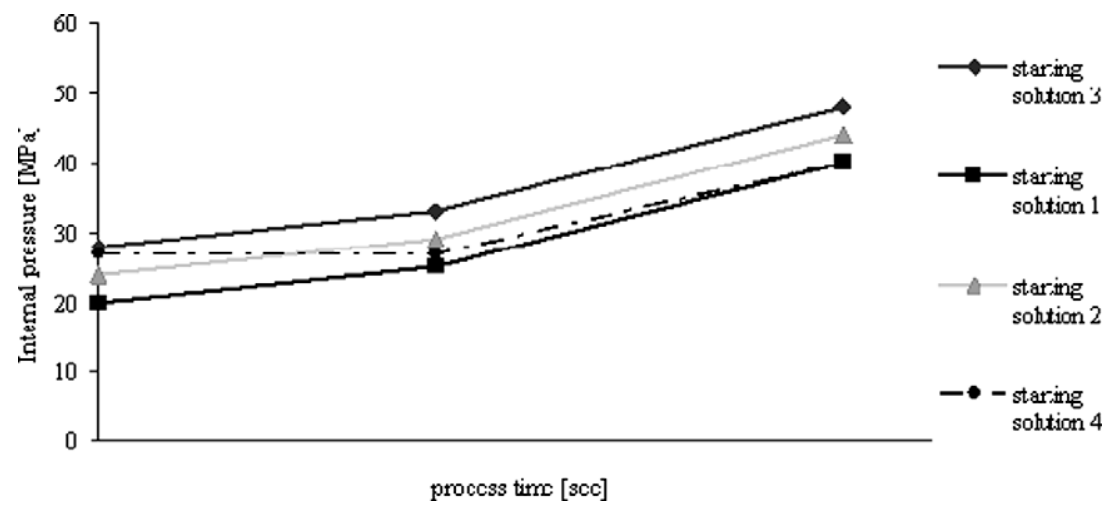

Fig. 20. The utilised starting pressure curves. 


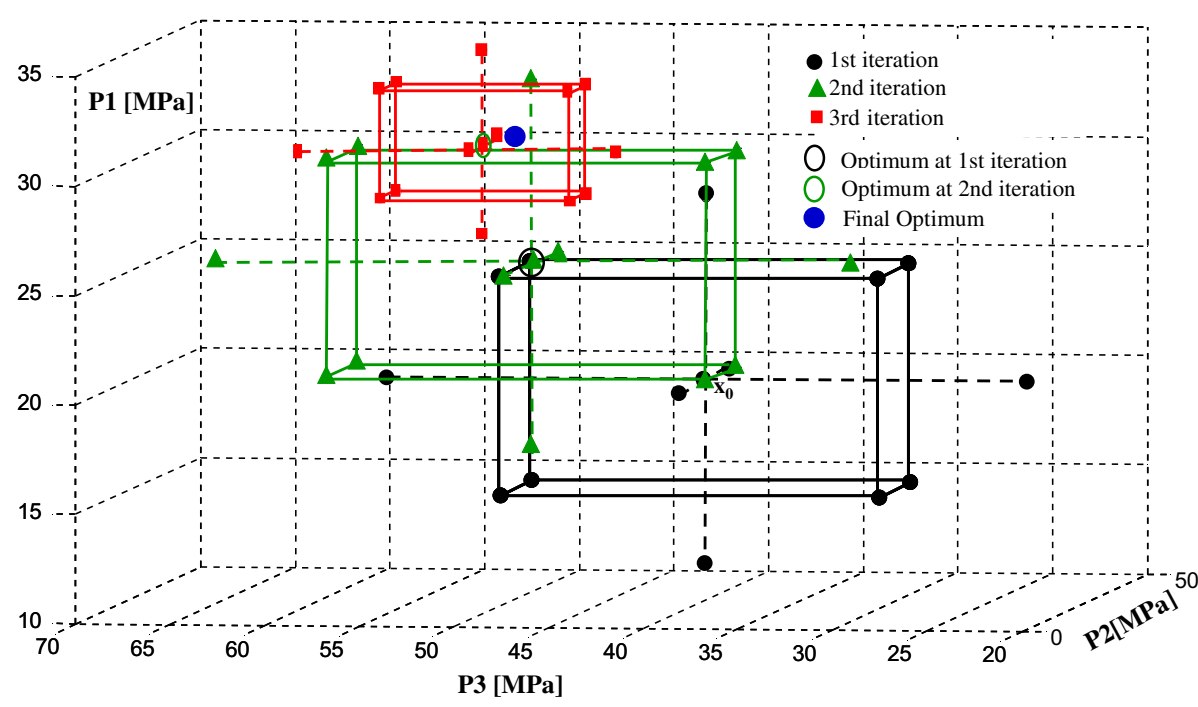

Fig. 23. The design space exploration with the fully second order approach.

As the latter issue above mentioned is concerned, the authors performed a wide analysis about the results obtainable by an approach based on a full exploration of the design space. In particular, the results obtained in case $2 \mathrm{~b}$ were utilised to perform the comparison. This approach was followed in order to evaluate the performances of the proposed cascade procedure in terms of optimal solution prediction and reduction of the number of direct problems to be investigated.

To this aim the procedure illustrated in Fig. 22 was developed. In particular, a Central Composite Design [18,41] was utilised to define a DOE over the whole design space. Of course, the dimensions of the design space were fixed since technological limits have to be respected: the widest technologically possible variations of the design variables (pressure values) were explored. On the explored design space, a RSM (based on the classical least squares approach) was utilised to approximate the response function for the chosen objective function using a second order approximation (it has to be noticed that the CCD is considered very appropriate to build up second order response surfaces). The first DOE was designed around the same initial solution used in case2b of the cascade procedure in order to compare the results at the same conditions. The objective function was evaluated within the points belonging to this design space and its minimum was searched within the same space by a sequential quadratic programming (SQP) approach. Starting from this minimum point, and, in particular, using such point as the new centre point of a new CCD, the procedure was iterated.

Thus, a second design space was explored (see Fig. 23), which was built like the first one, but around the previous optimum found. Successively, a new updated optimum was determined and a new search domain was defined around it. In particular, a width of the DOE which was half of the one previously utilised was chosen, in order to explore the design space locally more in detail. During the progression of the procedure, the region of interest moves and zooms on each optimum, in order to make the influence region more and more concentrated around the optimum. It is worth pointing out that at each iteration of the procedure validation and testing steps of the obtained response surfaces were developed; moreover, very performing values of the $R_{\text {adj }}^{2}$ (correlation index related to the prediction performances of the obtained response function) were obtained for each step. The iterations of such optimisation procedure (see again Fig. 23) led to the same optimal values of the design variables reached by the cascade procedure, after the solution of 45 direct problems (numerical simula- tions). The number of numerical simulations developed along the cascade procedure was 20.

This result can be summarised in the following issues:

1. In order to validate the cascade approach a comparison with a different (fully second order) procedure was developed;

2. the design space was explored more in details by the fully second order approach proving that the proposed cascade procedure does not neglect different optimum regions;

3. the total number of numerical simulations to be run to implement a fully second order approach is higher than the one required for the cascade procedure; nevertheless the same optimum was reached proving the effectiveness of the cascade approach, in particular with respect to the computational effort.

\section{Conclusions}

A cascade optimisation procedure was implemented in order to optimise a complex hydroforming operation aimed to produce Y-shaped tubes. The knowledge base available on tube hydroforming optimisation was utilised to assess some basic hypotheses on the investigated process, thus the internal pressure path was optimised taking into account two different optimisation cases.

The optimisation procedure integrates steepest descent method and response surface approach based on moving least squares approximation in order to reach a fine-tuning of the optimal solution. The approach seems very effective since it proved its ability to reach solutions which improve final part quality.

The procedure is very fast and performing since it exploits the advantages of both the integrated procedures. In fact, a few direct problems were necessary to reach the optimum also due to the idea to reuse, for MLS implementation, points of the design variables domain already available from steepest descent procedure iterations.

The reached solution provides very satisfactory effects from a technological point of view, leading to a final component with no fracture and a good shape in the bulged zone.

Thus, such optimisation strategy could be very efficient as design tool for industrial components production. Moreover, a sensitivity analysis on the starting solution proved that the obtained optimum can be considered a robust solution. What is more, the comparison with a fully second order approach provided a very effective validation of the cascade approach. 
The main conclusions on the proposed cascade procedure can be summarised as follows:

- an optimisation strategy based on a first order approximation (steepest descent method) by a second order one (based on MLS approximation) in the neighbours of the minimum is a very performing procedure to reach a high quality Y-shaped tube by optimising pressure action along an hydroforming operation;

- the results obtained on the final component are very satisfying in comparison with a gradient approach;

- the idea to explore the design space step by step, zooming around the expected minimum by a gradient technique before determining an optimal value through a second order approach was validated by comparing it with a fully second order procedure that led to the same results but with an increased computational effort;

- the MLS ability to manage irregular grid of data also contributed to lessen the necessary numerical simulations number since some of the explored design space points were reused to approximate the response function by MLS itself.

\section{References}

[1] Meinders T, Burchitza IA, Bonte MHA, Lingbeek RA. Numerical product design: springback prediction, compensation and optimization. Int J Mach Tools Manuf 2008;48:499-514.

[2] Fann K, Hsiao P. Optimization of loading conditions for tube hydroforming. J Mater Process Technol 2003;140(1-3):520-4.

[3] Kleinermann J, Ponthot J. Parameter identification and shape/process optimization in metal forming simulation. J Mater Process Technol 2003;139(1-3):521-6.

[4] Ponthot J, Kleinermann J. Optimisation methods for initial/tool shape optimisation in metal forming processes. Int J Vehicle Des 2005;39(12):14-24

[5] Naceur HA, Batoz J, Guo Y, Knopf-Lenoir C. Optimization of drawbead restraining forces and drawbead design in sheet metal forming process. J Mater Process Technol 2004;146:250-62.

[6] Jirathearanat S, Altan T. Optimization of loading paths for tube hydroforming. In: Proceedings of NUMIFORM, Columbus, OH, USA; 2004

[7] Lin Z, Juchen X, Xinyun W, Guoan H. Optimization of die profile for improving die life in the hot extrusion process. J Mater Process Technol 2003;142(3):659-64

[8] Zhao G, Ma X, Zhao X, Grandhi R. Studies on optimization of metal forming processes using sensitivity analysis methods. J Mater Process Technol 2004:147:217-28.

[9] Schenk O, Hillmann M. Optimal design of metal forming die surfaces with evolution strategies. Comput Struct 2004;82:1695-705.

[10] Abedrabbo N, Zafar N, Averill R, Pourboghrat F, Sidhu R. Optimization of a tube hydroforming process. In: Proceedings of NUMIFORM, Columbus, OH, USA; 2004.

[11] Poursina M, Antonio C, Parvizian J, Sousa L, Castro C. Eliminating folding defect in forging parts using a genetic algorithm. In: Proceedings of ESAFORM, Salerno, Italy; 2003.

[12] Do T, Fourment L, Laroussi M. Sensitivity analysis and optimization algorithms for $3 \mathrm{D}$ forging process design. In: Proceedings of NUMIFORM, Columbus, $\mathrm{OH}$, USA; 2004.

[13] Fourment L, Do T, Habbal A, Bouzaiane A. Gradient non gradient and hybrid algorithms for optimizing 2D and 3D forging sequences. In: Proceeding of ESAFORM, Cluj-Napoca, Romania; 2005.

[14] Castro C, Antonio C, Sousa L. Optimisation of shape and process parameters in metal forging using genetic algorithms. J Mater Process Technol 2004;146:356-64.

[15] Jansson M, Nilsson L, Simonsson K. On process parameter estimation for the tube hydroforming process. J Mater Process Technol 2007;190:1-11.

[16] Sheng Z, Jirathearanat S, Altan T. Adaptive FEM simulation for prediction of variable blank holder force in conical cup drawing. Int J Mach Tools Manuf 2004;44:487-94

[17] Labergere C, Gelin JC. New strategies for optimal control of command laws for tube hydroforming processes. In: Proceedings of NUMIFORM, Columbus, $\mathrm{OH}$, USA; 2004.
[18] Myers RH, Montgomery DC. Response surface methodology process and product optimization using designed experiments. 2nd ed. New York (USA): John Wiley and Sons, Inc.; 2002.

[19] Breitkopf P, Naceur H, Rassineux A, Villon P. Moving least squares response surface approximation: formulation and metal forming applications. Comput Struct 2005;83:1411-28.

[20] Jansson T, Andersson A, Nilsson L. Optimization of draw-in for an automotive sheet metal part-an evaluation using surrogate models and response surfaces. J Mater Process Technol 2005;159:234-426.

[21] Naceur H, Ben-Elechi S, Knopf-Lenoir C, Batoz J. Response surface methodology for the design of sheet metal forming parameters to control springback effects using the inverse approach. In: Proceedings of NUMIFORM, Columbus, $\mathrm{OH}$ USA; 2004.

[22] Naceur H, Guo YQ Ben-Elechi S. Response surface methodology for design of sheet forming parameters to control springback effects. Comput Struct 2006;84:1651-63.

[23] Buranathiti T, Cao J, Baghdasaryan L, Chen W, Xia C. Approaches for mode validation: methodology and illustration on a sheet metal flanging process. ASME J Manuf Sci Eng 2006;128:588-97.

[24] Oudjene M, Ben-Ayed L, Delameziere A, Batoz JL. Shape optimisation of clinching tools using the response surface methodology with Moving LeastSquare approximation. J Mater Process Technol 2009;209:289-96.

[25] Gurlebeck K, Konke C. Adaptive response surface approach using artificial neural network and moving least squares. In: Proceedings of seventeenth international conference on the application of computer science and mathematics in architecture and civil engineering, Weimar, Germany; 12-14 July, 2006.

[26] Belytschko T, Krongauz Y, Organ D, Fleming M, Krysl P. Meshless method: an overview and recent development. Comput Methods Appl Mech Eng 1996;139:3-47.

[27] Belytschko T, Lu YY, Gu L. Crack propagation by element-free galerkinmethods. Eng Fract Mech 1995;51(2):295-315.

[28] Most T, Bucher C. An enhanced moving least squares interpolation for the element-free galerkin method III. In: Proceedings of European conference on computational mechanics solids, structures and coupled problems in engineering, Lisbon, Portugal; 5-8 June, 2006.

[29] Rassineux P, Villon Breitkopf P. Simultaneous surface and tetrahedron mesh adaptation using meshfree techniques. Int $\mathrm{J}$ Numer Methods Eng 2003;57:371-89.

[30] Ahmetoglu M, Altan T. Tube hydroforming: state-of-the-art and future trends. J Mater Process Technol 2000;98:25-33.

[31] Yang J, Jeon BS. Design sensitivity analysis and optimization of hydroforming process. J Mater Process Technol 2001;113:666-72.

[32] Zadeh HK, Mashhadi MM. Finite element simulation and experiment in tube hydroforming of unequal $\mathrm{T}$ shapes. J Mater Process Technol 2006;177: 684-7.

[33] Imaninejad M, Subhash G, Lokus A. Loading path optimization of tube hydroforming process. Int J Mach Tools Manuf 2005;45:1504-14.

[34] Ray P, Mac Donald BJ. Determination of the optimal load path for tube hydroforming processes using a fuzzy load control algorithm and finite element analysis. Finite Elem Anal Des 2004;41:173-92.

[35] Manabe K, Suetake M, Koyama H, Yang M. Hydroforming process optimization of aluminium alloy tube using intelligent control technique. Int J Mach Tools Manuf 2006;46:1207-11.

[36] Di Lorenzo R, Ingarao G, Micari F. Process parameters calibration in 3D tube hydroforming processes. In: Proc. Esaform'07; 2007. p. 411-6.

37] Di Lorenzo R, Ingarao G, Gagliardi F, Filice L. Experimental validation of optimisation strategies in hydroforming of T-shaped tubes. In: Proceeding Esaform'08; 2008

[38] Jirathearanat S, Hartl C, Altan T. Hydroforming of Y-shapes product and process design using FEA simulation and experiments. J Mater Process Technol 2004;146:124-9.

[39] Ponthot JP, Kleinermann J. A cascade optimization methodology for automatic parameter identification and shape/process optimization in metal forming simulation. Comput Methods Appl Mech Eng 2006;195:5472-508.

[40] Di Lorenzo R, Ingarao G, Chinesta F. A gradient-based decomposition approach to optimize pressure path and counterpunch action in Y-shaped tube hydroforming operations. Int J Adv Manuf Technol 2008. doi:10.1007 s00170-008-1813-x [Springer-Verlag London Limited].

[41] Montgomery DC. Design and analysis of experiments. 4th ed. New York: Wiley; 1997

[42] Doltsinis I. Inelastic deformation processes with random parameters methods of analysis and design. Comput Methods Appl Mech Eng 2003;192:2405-23.

[43] Ingarao G, Di Lorenzo R, Micari F. Internal pressure and counterpunch action design in Y-shaped tube hydroforming processes: a multi-objective optimisation approach. Comput Struct 2009;87:591-602. 